\title{
Developing Data Logger with Industrial IoT for Gas Station
}

\author{
Jeongseok $\mathrm{Oh}^{1}$ and Byunghun Song ${ }^{2}$ \\ ${ }^{1}$ Institute of Gas Safety R\&D, Korea Gas Safety Corporation, 1390, Wonjung-ro, \\ Maengdong-myeon, Emseong-gun, Chungcheongbuk-do, Korea \\ ${ }^{2}$ Smart Factory ICT $R \& D$ Center/Convergence Industries $R \& D$ Division, Korea \\ Electronics Technology Institute, \#22, Daewangpangyo-ro 721beon-gil, \\ Bundang-gu, Seongnam-si, Gyeonggi-do, Korea \\ 1jsoh90@gmail.com, ${ }^{2}$ bhsong@keti.re.kr
}

\begin{abstract}
As wireless communication technology advances, industrial fields have been adopted for efficient management. It is very dangerous that industrial fields try to apply generous wireless method without reliability and safety requirement. Especially, industrial gas safety fields are necessary more reliability and safety because of correlation between gas accident and wireless communication method. This paper aims to develop practical use technology of remote wireless sensing system for gas facilities. At first, we evolve network management and connection for gas facilities in order to find, control and manage gas devices with identification code. Next, our study design high reliability network adaptor with heterogeneous gas devices. Our research develop data logger as a sensor node. This data logger is divided into A type and B type with its intended use. Finally, we construct two test-beds in order to validate our results in field.
\end{abstract}

Keywords: wireless, risk parameters, gas data transmitter, gas data logger

\section{Introduction}

In general, industrial gas safety management for gas facilities should be considered more carefully because those facilities have been operated by various kinds of materials. In gas accidents, human is damaged directly because most type is explosion, fire and rupture. Especially, gas station, gas storage tank and gas pipeline have showed frequently diverse accidents, because they are seemed to satisfy only institutional requirements. As wireless communication technology advances, industrial fields have been adopted for efficient management. It is very dangerous that industrial fields try to apply generous wireless method without reliability and safety requirement. Especially, industrial gas safety fields are necessary more reliability and safety because of correlation between gas accident and wireless communication method.

This paper aims to develop practical use technology of remote wireless sensing system for gas facilities. At first, we evolve network management and connection for gas facilities in order to find, control and manage gas devices with identification code. The optimized identification system is for gas facilities are designed by considering the whole function of system and characteristic of device. Furthermore, this research design data format of source identification in gas device. Next, our study design high reliability network adaptor with heterogeneous gas devices. Our design concerns sensor node ds and gateway for remote monitoring and control on gas facilities. Designing our devices aim to define functions of sensor node and gateway. Designing our sensor node aims to low power consumption, reusability

Received (August 16, 2017), Review Result (October 10, 2017), Accepted (October 16, 2017) 
and distributable ability on account of adding legacy system. Also, gateway architecture should be divided roughly into PHYs layer, Kernel layer, APIs layer, APP layer in order to satisfy independent function. Next, we develop practical package about data logger for gas facilities. Our research develop data logger as a sensor node. This data logger is divided into A type and B type with its intended use. A type does functions like as control response message of gas circuit breaker, status information request message of breaker, image registration request message, and sending sensor values to gateway. B type of data logger is designed as shockproof and waterproof for operating stably and adapting various environmental factors. Finally, we construct two test-beds in order to validate our results in filed. The first test-bed is small gas plant that may charge various gases into tank and sell the use of gas. The second test-bed is small storage gas tank in order to provide LPG about twenty families.

\section{Developing Network Management and Connection for Gas Facilities}

\subsection{Developing Identification Code for Finding, Controlling and Managing Data Logger}

OID (Object Identifier) is defined as distinct value that can differentiate objects in general. Because the use of past identification code is insufficient to meet current global operation system with convergence between IT and legacy industry, designing systematic identification system is necessary in order to manage efficiently and stably. Thus, this research is to design OID system for optimizing gas facilities. The optimized identification system for gas facilities are designed by considering the whole function of system and characteristic of device. Also, each item in identification code can be select over one value, and then sub-item may select different value depending on the related item value. Major item are described as Table 1 .

\section{Table 1. Major Item}

\begin{tabular}{|l|l|}
\hline Item name & Item Description \\
\hline Country & $\begin{array}{l}\text { The indication of nation is allocated by ISO } \\
\text { Ex) 1.2.4.10 : this item value means OID of Korea }\end{array}$ \\
\hline $\begin{array}{l}\text { Registered } \\
\text { corporation }\end{array}$ & $\begin{array}{l}\text { The indication of nation is allocated by ISO 02:00:00 } \\
\text { Ex) 1.2.410.200060: this item value means OID of Korea gas } \\
\text { corporation }\end{array}$ \\
\hline Bridge & $\begin{array}{l}\text { This item indicates bridge } \\
\text { 1: server, 2: gateway } \\
\text { Ex)1.2.140.200060.1.1.2: this item value means gateway OID of Korea } \\
\text { gas corporation }\end{array}$ \\
\hline Bridge Serial & $\begin{array}{l}\text { This item indicates serial number of bridge } \\
101 \sim 103,201 ~ 203,301 ~ 303,401 ~ 403 \\
\text { Ex) 1.2.410.2000060.1.1.2.101 this item value means OID of } 101 \\
\text { serial number in gateway }\end{array}$ \\
\hline $\begin{array}{l}\text { Extension } \\
\text { node }\end{array}$ & $\begin{array}{l}\text { 1: sensor node } \\
\text { Ex) 1.2.410.2000060.1.1.2.10.1 this item value means OID of sensor } \\
\text { node }\end{array}$ \\
\hline $\begin{array}{l}\text { Extension } \\
\text { device }\end{array}$ & $\begin{array}{l}\text { This item indicates device at extension node } \\
1: \text { sensor, 2: actuator } \\
\text { Ex) 1.2.410.2000060.1.1.2.10.1.1001.1 this item value means OID of } \\
\text { sensor at sensor node }\end{array}$ \\
\hline
\end{tabular}




\begin{tabular}{|l|l|}
\hline Extension & This item indicates type of extension device \\
node & 10: default, 11: gas sensor, 12: temperature sensor, 13: pressure sensor \\
& 14: corrosion sensor, 15: vibration sensor, 16: weight sensor \\
& 17: windvelocity sensor, 18: humidity sensor, 19: ultraviolet sensor \\
& 20: motion sensor, 21: illumination sensor, 22: radioactivity sensor \\
& 23: noise sensor, 24: co2 sensor, 25: gps sensor(lat), 26: gps \\
& sensor(lot) \\
& 27: image sensor, 28: strain sensor, 29: incline sensor, 30: waterhigh \\
& sensor \\
& 31: watertemp sensor, 32: windvane sensor \\
& Ex) 1.2 .410 .2000060 .1 .1 .2 .10 .1 .1001 .1 .12 this item value means OID \\
& of temperature sensor type at sensor node \\
\hline
\end{tabular}

Furthermore, this research is to design data format of source identification in gas device. At first, we define communication message format between sensor node and gateway. Message is divided into Header and Body, and all messages have Header of same type. In example, request format is shown as Table 2.

Table 2. Request Message Format

\begin{tabular}{|l|l|l|l|}
\hline Header & \multicolumn{2}{|l|}{ Request Message } \\
\hline Total Length & $\mathbf{7 4}$ & Length & Comment \\
\hline NO & Item & 4 & Total length except data length \\
\hline 1 & Data length & 4 & Protocol message \\
\hline 2 & Message Code & $\begin{array}{l}\text { Separation of message sequence } \\
\text { this value can have from 0 to } \\
\text { 65535) }\end{array}$ \\
\hline 3 & $\begin{array}{l}\text { Sequenced number of } \\
\text { message request }\end{array}$ & 2 & Object identifier \\
\hline 4 & OID & 26 & NULL when connecting BLE \\
\hline 5 & MAC address & 18 & NULL when connecting BLE \\
\hline 6 & IP address & 16 & 100 \\
\hline 7 & Header division & 4 & \\
\hline
\end{tabular}

Next, this research would define OID format of HTTP communication protocol between gateway and server. This communication does not distinguish Header and Body like as Table 3. Gateway send message to server using GET_POST. In other way, sever send message to server using Json. Gateway send OID information and server send short message without unnecessary information.

Table 3. Get Post Message Format

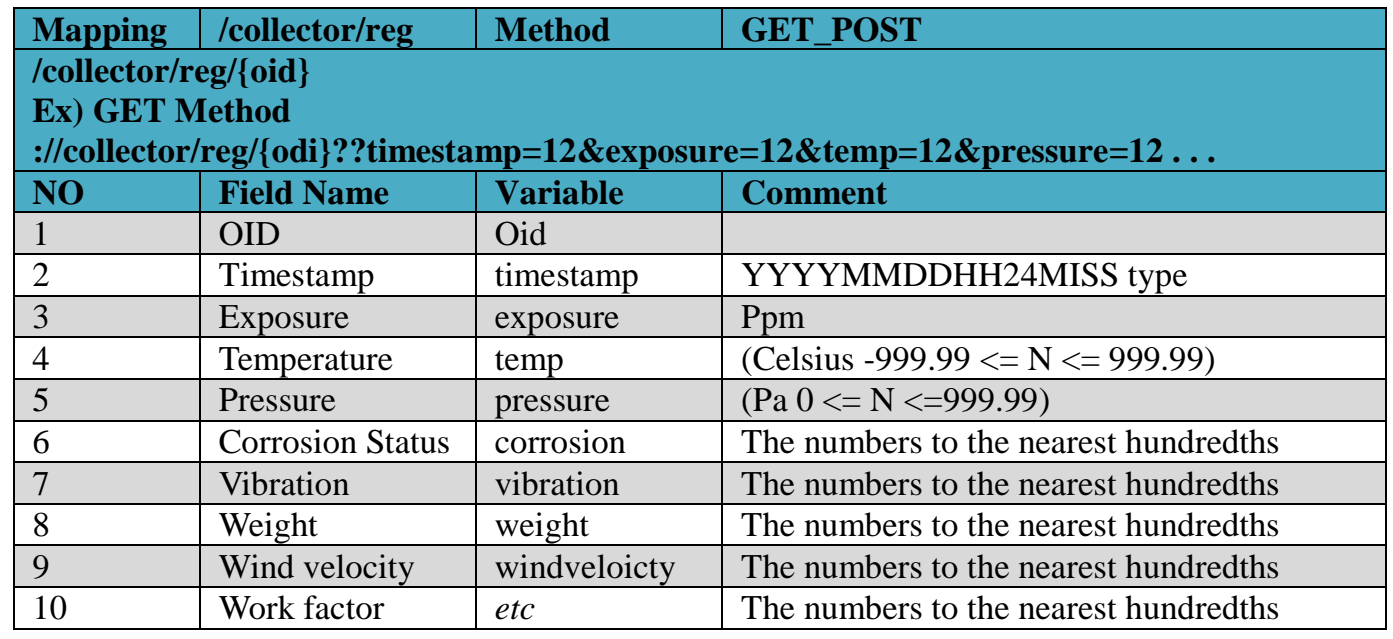




\subsection{Designing Data Logger as High Reliability Network Adaptor}

Our design concerns sensor node for remote monitoring and control on gas facilities. Designing our sensor node aims to low power consumption, reusability and distributable ability on account of adding legacy system. Wireless communication between sensor node and gateway has $1: \mathrm{N}$ relation using Wi-Fi, BLE or Wi-SUN, otherwise communication between gateway and server has M:N relation. Sensor nodes must be installed on proper location for gathering related status data of risk analysis in gas facilities. A gateway would communicate district sensor nodes for managing gas facilities, and play a role as repeater for sending message to server or receiving message from server. A server manages remotely gateway and sensor nodes. Especially, functions of sensor node are followings

- Data collection function from sensor module: This function collects repeatedly data form sensor modules, and saves average values in memory. In case of vibration, this study saves checking vibration value between time intervals in interrupt using change of pine value.

Message transmit/receive function of gateway: when one side send request message, the other side send response message. This function must have filtering function to lost data in order to protect receive communication error, and this can transmit response message by adding status of request message

- Message transmit function to gateway: this function should integrate three functions to a message according to protocol. Thus information is as followings. The first information is data from gathering sensor module. The next is alarm to control of gas leak breaker by interrupt. The third is response toward control of gas circuit breaker by requesting of gateway.

Message receive function to gateway: this function convert three functions that are received from gateway to a message according to protocol. Thus information is as followings. The first information is response message to transmission of sensor data. The next is response message to alarm of breaker control by interrupt. The third is request message for control of gas circuit breaker from gateway. If sensor node receives request message for control of gas circuit breaker, it will control gas circuit breaker and transmit response message.

- Lost data filtering function: this function would filter failure data by checking whether the received data is lost data or not.

- Control function of gas circuit breaker: this function is able to control gas circuit breaker. Gas circuit breaker can be controlled by itself or by requesting of gateway.

- Control function of gas circuit breaker by interrupt of sensor node: sensor node may gather repeatedly sensor data. If interrupt occur out of threshold range of gas leak, the senor node can activate gas circuit breaker. Also, sensor node notifies change of status to gateway when activating gas circuit breaker.

Control function of gas circuit breaker by request of gateway: gateway can activate indirectly gas circuit breaker through sensor node. The sensor node can activate or deactivate gas circuit breaker as request of gateway.

System architecture of gateway is divided roughly into PHYs layer, Kernel layer, APIs layer, APP layer like as Figure 1. The PHYs layer is implemented physical protocol such as Ethernet, Wi-Fi, Bluetooth bock for networking. Kernel layer have various functions to control hardware for operating gateway such as hardware management and network management. APIs layer is classified into kernel module and libraries. Kernel module has a play role as connecting organically between hardware and software for supporting network on OS such as IPv6, IPv4 and 
security. Libraries support partially necessary function in software which consists of APIs and Elements. APP layer is management on software for operating. Control management is ability for operating gateway function with connecting APIs layer. Data management maintains transmission, receives data through network and provides information to control management.

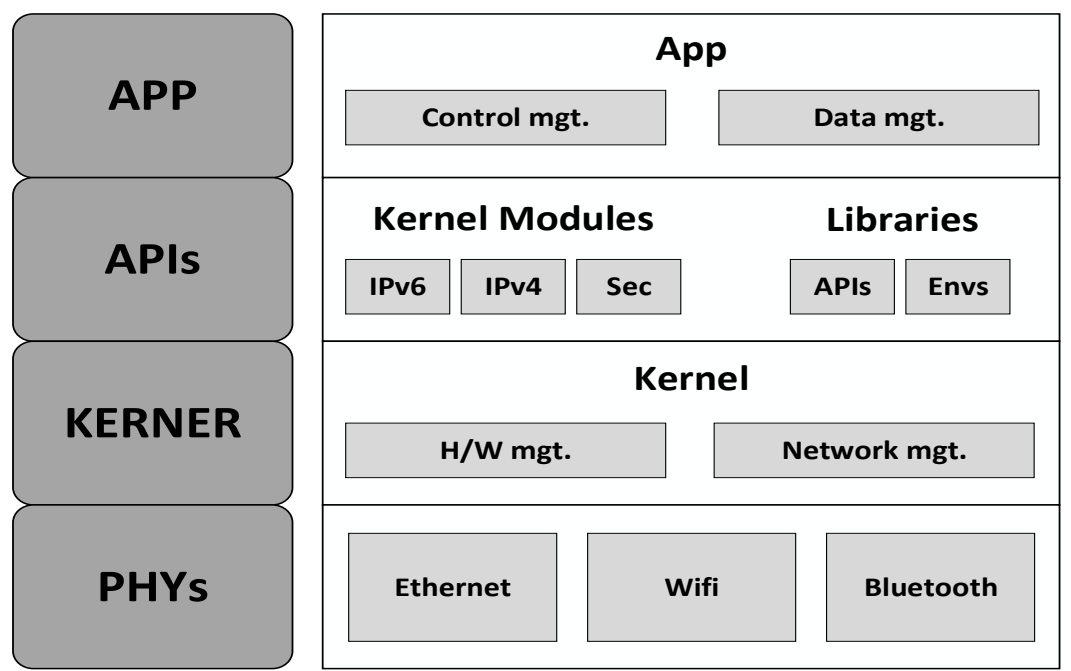

Figure 1. System Architecture and Function

In our research, functions of gateway are followings.

Receiving request message to registration sensor information from sensor node and transmitting response message to registration sensor information of sensor node: sensor nodes would gather the measured data in sensor such as gas exposure, temperature, humidity and vibration, and then they send this information to gateway. A gateway receives message with these information. A gateway checks message status and responses message with check information to sensor nodes after it received sensor information.

Receiving request message for registration sensor information of gas circuit breaker and transmitting response message to registration sensor information of gas circuit breaker: A sensor node can connect directly gas circuit breaker in order to receive heterogeneous information. Especially, a sensor node is ability for checking status of gas circuit breaker and sends the checked information to gateway. A gateway checks message status and responses message with check information to sensor nodes after it received sensor information.

Receiving request message to operate gas circuit breaker and transmitting response message to operate gas circuit breaker: A gateway can send control message such as block, unblock and retaining state for operating gas circuit breaker to sensor node. A sensor node send response message with status information to a gateway.

- Transmitting request message for registration sensor information to server and receiving response message to registration sensor information from server: A gateway collect sensor information, and then send information per sensor node to server. A server checks message status and responses message with check information to gateway.

- Transmitting request message for registration sensor information of gas circuit breaker to server and receiving response message to registration sensor information of gas circuit breaker from server: A gateway collect status information of breaker through sensor node, and then send information to server. When as server checks status 
information and add status such as normal or abnormal to response message, a gateway receive this message.

Receiving request message to operate gas circuit breaker from server and transmitting response message to operate gas circuit breaker: a server can create message control message for operating breaker in case of gas leakage and a gateway receive this message. Also, a gateway deliveries control message to sensor node and responses message whether breaker is operated successfully or not.

Filtering lost data: a gateway check the validity of received message from many sensor nodes and a server with connection a gateway may. If the message is not available, a gateway can filter it

- Creating RESTful Web data: various information that is managed by gateway converts into RESTful web data type. This web data may support open web service through server.

Saving data: a gateway save values about operating status and environmental configuration from receiving sensor nodes and server. The saved data is used by maintaining and repairing device and deleted automatically after the specified interval.

Blocking connection: If a gateway can block interface connection by discontinuity of network connection or failure of internal operation.

\section{Developing Data Logger Package with Industrial IoT}

Our research develop data logger as a sensor node. This data logger is divided into A type and B type with its intended use. A type of data logger communicates simultaneously ten sensor modules using analog pin in gas, vibration, and ultraviolet sensors and digital pin in PIR motion sensor. I2C communication is used in measuring temperature, humidity and pressure, and use bus individually because of connecting parallel. Latitude and longitude of Information of GPS uses serial communication, and photo sensor gets image information through RS 232. A type does functions like as control response message of gas circuit breaker, status information request message of gas circuit breaker, image registration request message, and sending sensor values to gateway. Figure 2 is flowchart of A type and describes the detailed contents by classifying function per each module.

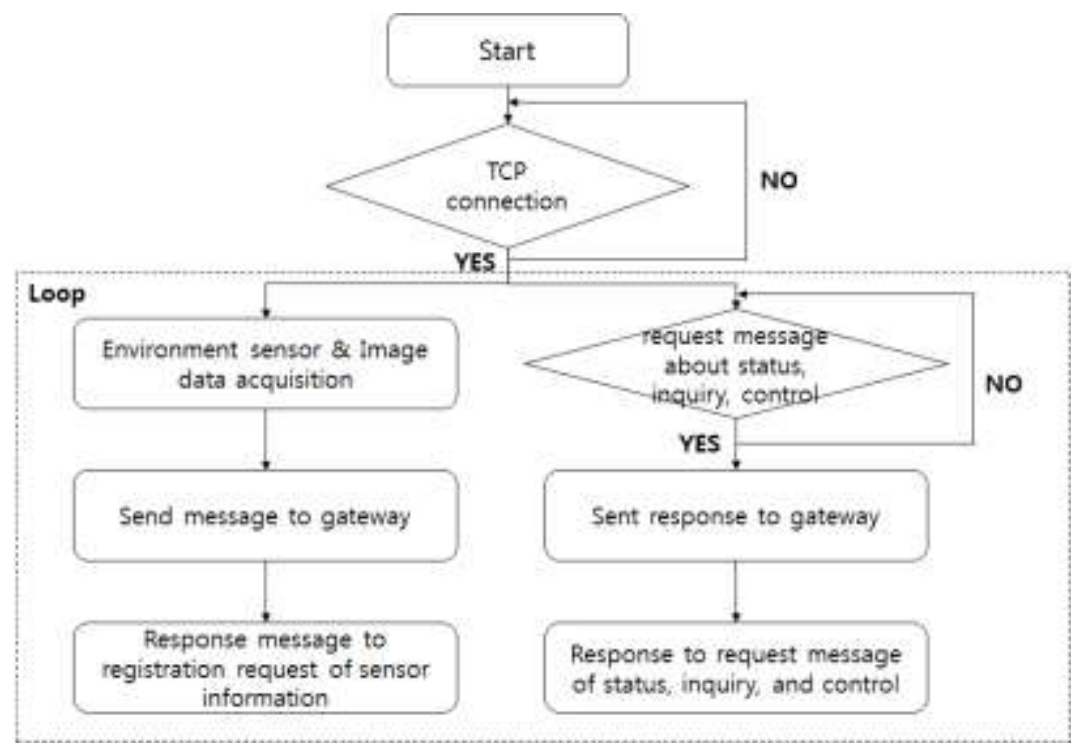

Figure 2. Flowchart of a Type Logger 
A type of logger reads basic information file for requiring TCP connection to gateway and carries out pairing for Wi-Fi communication by means of the initial configuration. This logger is operated roughly by collecting data, transmitting data, and receiving message by requesting of gateway. The transmission/receive subroutine is activated as thread in order to communicate environmental sensor data to gateway. Collecting environmental sensor data is send at regular intervals, and those intervals can change through environmental configuration file. A type of data logger is capable of gathering various sensor data such as gas, temperature, humidity, pressure, vibration, ultraviolet rays, PIR motion, latitude and longitude. The A type gateway comprises a programmable board and various modules via communication interfaces. The board contains a $720 \mathrm{MHz}$ ARM Cortes A8 processor, SDRAM 256MB, and NAND Flash 256 MB. Embedded Linux is installed as an operating system. Additional SD memory in the board is utilized for logging an exceptional event and transmitted data and for updating the system. Power is provided by converting AC $220 \mathrm{~V}$ to $\mathrm{DC} 5 \mathrm{~V}$ as the input voltage. If an unexpected problem in the wired power supply occurs, an internal subsidiary battery module provides input voltage. The gateway performs communication functions with the sensor nodes by Bluetooth 4.0 (Bluetooth low energy [BLE]), so it can be simultaneously connected with several sensor nodes. Additionally, it interacts with servers over TCP and HTTP via a 3G/LTE modem or internal Ethernet.

$\mathrm{B}$ type of data logger is designed as shockproof and waterproof for operating stably and adapting various environmental factors. This data logger type can communicate reliably by means of adding Wi-Fi antenna. This logger has various functions as followings.

Reading data from sensor and camera module: This type can read data from each sensor module through various interfaces. Collection data may be temperature, humidity, carbon dioxide, atmospheric pressure, noise, gas, radioactivity, latitude, longitude, vibration, motion, intensity of illumination, still image, and so on.

Transmitting sensor information to gateway: this type must change various sensor data into message of packet mode, and then send message to gateway.

- Communicating message between gateway and data logger: data logger and gateway should send request message and receive response message as occasion demands.

- Giving on/off command for operation gas circuit breaker: when specific situation such as gas leak arise, gateway can order operation of breaker through data logger with communicating on/off message.

IDE of this type is operated based on setup and loop function. At first, setup function works various settings for requiring system operation like as figure 3 . The main board of this logger should carry out monitoring and debugging using hardware serial because it is not able to take OS porting. 


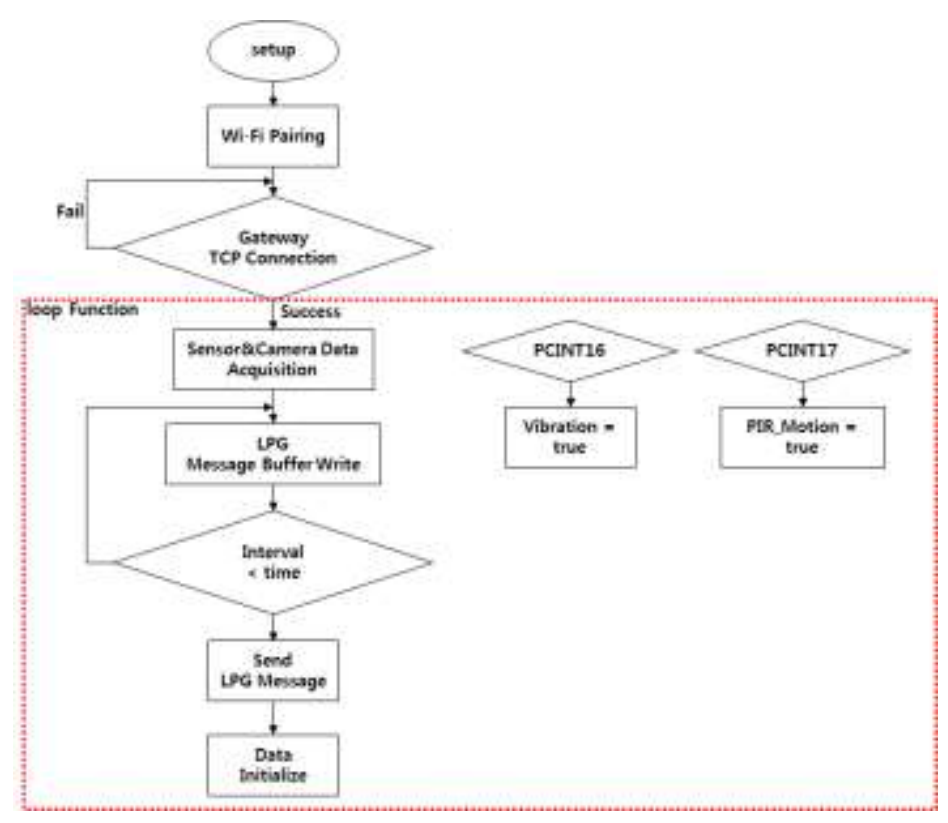

Figure 2. Flowchart of B Type Logger

Loop function is classified into three parts that are performed repeatedly after initialization. The first part is collecting data from each sensor module. The next is sending sensor data to gateway. The last part is receiving request and response message from gateway. The B type gateway is made of a Raspberry Pi 2 Model B. The Raspberry Pi 2 Model B is a suitable board as a gateway in a limited environment because it contains a $900-\mathrm{MHz}$ quad-core ARM Cortex-A7 processor and SDRAM $1 \mathrm{~GB}$; it demonstrates a high performance for data processing and communications despite being tiny. It runs embedded Linux as an operating system and receives and transmits data with sensor nodes like the A-type gateway. The communication function between the gateway and sensor node is reliably carried out over TCP via the Ethernet. Therefore, the gateway has the advantage of being able to interconnect with many more sensor nodes compared to Bluetooth 4.0 (BLE) in the A-type gateway. It also interacts with servers over TCP and HTTP via the Ethernet.

\section{Field Demonstration Test by Constructing Test-Bed}

This research construct two test-beds in order to test our results in filed. The first test-bed is small gas plant that may charge various gases into tank and sell the use of gas. This plant is consists of four storage tanks, four charging places, and three supply room. Four storage tanks should preserve liquefied oxygen(20.5 ton), liquefied argon(16.4 ton), liquid nitrogen(10.9 ton), and liquefied carbon(25.0 ton) respectively. Three supply rooms preserve cylinders that are filled in high-pressure gas, LPG, and inflammable gas respectively. In this test-bed, A type of data loggers are installed at the cylinder support room for sensing LPG and environmental information. B type of data loggers are installed at ceiling of gas tank for monitoring pressure gauge. For monitoring pressure of liquefied oxygen, liquefied argon, and liquid nitrogen, we install three optic photo sensors respectively. A gateway that can gather information of A type and B type of data loggers is installed at rooftop of business facility. This gateway should send to server with integrating data. The second test-bed is small storage gas tank in order to provide LPG about twenty families. This facility have about 2.9 ton of gas tank and 50A size of pipelines. In this test-bed, both data logger type are installed around fence of small 
gas tank, and a gateway is installed beside gas tank. We install two optic photo sensors in order to monitor LPG capacity and pressure gauge. This information sends to sever through gateway.
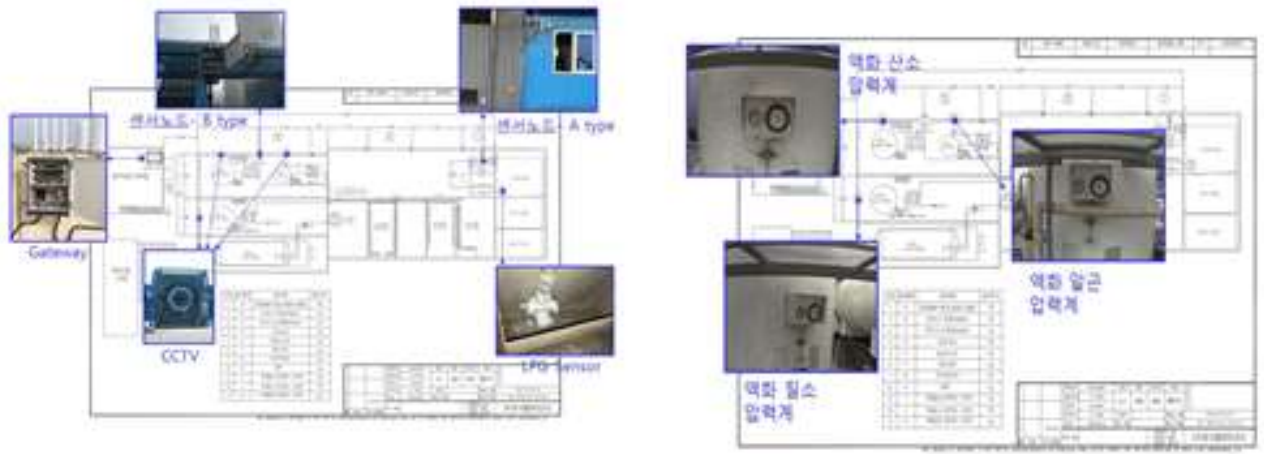

Figure 3. Location of Installed Devices in the First Test-bed

\section{Conclusions and Future Works}

In general, industrial gas safety management for gas facilities should be considered more carefully because those facilities have been operated by various kinds of materials. In gas accidents, human is damaged directly because most type is explosion, fire and rupture. Especially, gas station, gas storage tank and gas pipeline have showed frequently diverse accidents, because they are seemed to satisfy only institutional requirements. As wireless communication technology advances, industrial fields have been adopted for efficient management. It is very dangerous that industrial fields try to apply generous wireless method without reliability and safety requirement. Especially, industrial gas safety fields are necessary more reliability and safety because of correlation between gas accident and wireless communication method. Furthermore, devices such as sensor, sensor node and gateway for wireless communication are considered ad installed along with environmental characteristics.

This paper developed practical use technology of remote wireless sensing system for gas facilities. At first, we evolved network management and connection for gas facilities in order to find, control and manage gas devices with identification code. The optimized identification system is for gas facilities are designed by considering the whole function of system and characteristic of device. Furthermore, this research designed data format of source identification in gas device and OID format of HTTP communication protocol between gateway and server. Next, our study designed high reliability network adaptor with heterogeneous gas devices. Our design concerns sensor node and gateway for remote monitoring and control on gas facilities. Our sensor node has eight functions for our networking infrastructure. Designing our sensor node must include low power consumption, reusability and distributable ability on account of adding legacy system. Also, our proposed gateway has nine functions. This gateway architecture should be divided roughly into PHYs layer, Kernel layer, APIs layer, APP layer in order to satisfy independent function. Next, we developed practical package about data logger for gas facilities. Our research defined data logger as a sensor node. This data logger is divided into A type and B type with its intended use. A type does functions like as control response message of gas circuit breaker, status information request message of breaker, image registration request message, and sending sensor values to gateway. A type of data logger is capable of gathering various sensor data such as gas, temperature, humidity, pressure, vibration, ultraviolet rays, PIR motion, latitude and longitude. 
B type of data logger is designed as shockproof and waterproof for operating stably and adapting various environmental factors. This logger are four functions that are reading data from sensor and camera module, transmitting sensor information to gateway, communicating message between gateway and data logger, and giving on/off command for operation breaker. Finally, we construct two test-beds in order to validate our results in filed. The first test-bed is small gas plant that may charge various gases into tank and sell the use of gas. In this test-bed, A type of data loggers are installed at the cylinder support room for sensing LPG and environmental information. B type of data loggers are installed at ceiling of gas tank for monitoring pressure gauge. The second test-bed is small storage gas tank in order to provide LPG about twenty families. In this test-bed, both data logger type are installed around fence of small gas tank, and a gateway is installed beside gas tank. We install two optic photo sensors in order to monitor LPG capacity and pressure gauge.

\section{Acknowledgments}

This work was supported by the International Collaborative Energy Technology R\&D Program of the Korea Institute of Energy Technology Evaluation and Planning (KETEP) granted financial resource from the Ministry of Trade, Industry \& Energy, Republic of Korea (No. 20148530050120).

This work was supported by the Information \& Communication and Broadcasting R\&D Program of the Institute for Information \& Communications Technology Promotion (IITP) granted financial resource from the Ministry of Science and ICT, Republic of Korea (No. R-20160530-003936, Development of Quality Inspection System based on IIoT Platform for improving Quality in Small and Medium manufactures).

\section{References}

[1] M.I. Moussa and T. Eid, "Risk Management for Chlorine Producing Factory in Egypt", Australian Journal of Basic and Applied Science, vol. 1, no. 2, (2007), pp. 239-248.

[2] A. Daniel and J. F. Louvar, "Chemical Risk Analysis: Fundamentals with Applications", Prenice Hall, (1990).

[3] L. Atzori, A. Iera, and G. Morabito, "The Internet of Things: A Survey", Computer Networks, vol. 54, no. 15 , (2010), pp. 239-248.

[4] R. Cardell-Oliver, K. Settem, M. Kranz and K. Mayer, "A reactive soil moisture sensor network: Design and valuation", International Journal of Distributed Sensor Networks, vol. 1, no.2, (2005), pp.149-162.

[5] C. Bisdikian, "An Overview of the Bluetooth Wireless Technology", IEEE Communications Magazine, vol. 39, no.12, (2001), pp.86-94.

[6] Raspberry Pi 2 Model, B, http://www.raspberrypi.org/products/raspbetty-pi-2-moel-b.

[7] Arduino Uno, http://www.arduino.cc/en/Main/arduinoBoardUno

[8] V. Henrique, K. M. Celso, R. G. Ivan, C. F. Tiago and R. P. José, "Leak detection in petroleum pipelines using a fuzzy system", Journal of Petroleum Science and Engineering, vol. 49, no 3-4, (2005), pp 223238.

[9] A. M. Akib, N. Saad, and V. Asirvadam, "Pressure Point Analysis for Early Detection System", Proceedings of IEEE $7^{\text {th }}$ International Colloquium on Signal Processing and Its Applications, Penang, Malaysia, (2010), pp. 103-107.

[10] J. S. Oh, H. J. Bang and H. Ko, "An Empirical Study on Smart Safety Management Architecture for Gas Facilities in Korea”, INFORMATION-An International Interdisciplinary Journal., vol. 15, no.3, (2012), pp. 1107-1122.

[11] J. S. Oh, “An Analysis Intelligent Methodology for Finding Gas Leak Location”, INFORMATION-An International Interdisciplinary Journal, vol. 16, no. 3(B), (2013), pp. 3297-3302.

[12] J. S. Oh, "Safety Monitoring System Design for LPG Supply Facilities", The Asian International Journal of Life Sciences, no. 12, (2015), pp. 167-177. 


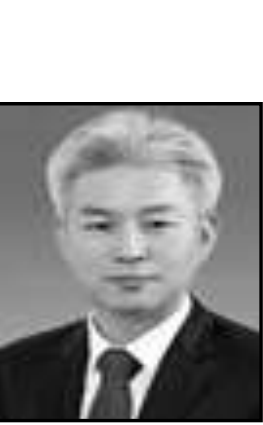

\section{Authors}

Jeong Seok Oh, he is a principal researcher at gas safety $R \& D$ institute in Korea Gas Safety Corporation. He received M.Sc degree and Ph.D degree both in computer science department from Soongsil University in 1998 and 2006 respectively. He has published more than 40 articles in convergence field between gas safety and IT, His main research interests include industrial IoT, risk analysis based on combination safety technology and information technology.

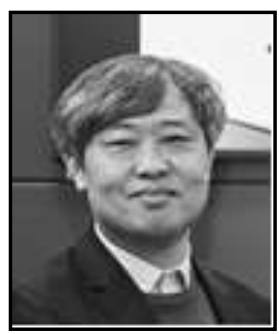

Byunghun Song, he is a head of Smart Factory ICT R\&D Center at Korea Electronics Technology Institute (KETI), Seongnam-si, South Korea. He received BS, MS and PhD degrees in electrical and electronic engineering from Kwangwoon University, South Korea, in 1998, 2000, and 2004 respectively. He serves as a leader of Infrastructure Team in Korea Smart Factory Foundation (KOSF) and a representative of OPC Foundation Korea. He has over 20 years of experience in the field of wireless sensor network and internet of things. He is now working in the Industrial Internet research center at KETI. His research interests and activities are in areas of Industrial IoT platform and infrastructure architecture for smart factory. 
International Journal of Control and Automation

Vol. 10, No. 12 (2017) 